\title{
Coherent interfaces with mixed hybridization govern direct transformation from graphite to diamond
}

Zhisheng Zhao ( $\nabla$ zzhao@ysu.edu.cn )

Yanshan University https://orcid.org/0000-0001-8492-0151

Kun Luo

Yanshan University

Bing Liu

Yanshan University

\section{Wentao Hu}

Center for High Pressure Science (CHiPS), State Key Laboratory of Metastable Materials Science and Technology, Yanshan University, Qinhuangdao 066004, China

\section{Xiao Dong}

Nankai University

\section{Yanbin Wang}

Center for Advanced Radiation Sources, The University of Chicago https://orcid.org/0000-0001-57163183

\section{Quan Huang}

Zhongyuan University of Technology

\section{Yufei Gao}

Yanshan University

Lei Sun

Yanshan University

\section{Yingju Wu}

Yanshan University

\section{Yang Zhang}

Yanshan University

\section{Mengdong Ma}

Yanshan University

\section{Xiang-Feng Zhou}

Yanshan University https://orcid.org/0000-0001-8651-9273

Julong He

Yanshan University

\section{Dongli Yu}

Yanshan University

Zhongyuan Liu 
Yanshan University

\section{Bo Xu}

Yanshan University https://orcid.org/0000-0002-9804-1614

\section{Yongjun Tian}

Yanshan University https://orcid.org/0000-0002-4594-4879

\section{Physical Sciences - Article}

Keywords: coherent graphite-diamond interfaces, graphite-to-diamond transformation, nanoscale coherent interfaces

Posted Date: November 9th, 2021

DOl: https://doi.org/10.21203/rs.3.rs-934066/v1

License: (1) (i) This work is licensed under a Creative Commons Attribution 4.0 International License. Read Full License

Version of Record: A version of this preprint was published at Nature on July 6 th, 2022. See the published version at https://doi.org/10.1038/s41586-022-04863-2. 


\section{Abstract}

Understanding the direct transformation from graphite to diamond has been a long-standing challenge with great scientific and practical importance. Previously proposed transformation mechanisms ${ }^{1-3}$, based on traditional experimental observations that lacked atomistic resolution, cannot account for the complex nanostructures occurring at graphite-diamond interfaces during the transformation ${ }^{4,5}$. Here, we report the identification of coherent graphite-diamond interfaces constituted with four structural motifs in partially transformed graphite samples recovered from static compression, using high-angle annular dark-field scanning transmission electron microscope. These observations provide vital insight into possible pathways of the transformation. Theoretical calculations confirm that transformation through these coherent interfaces is energetically favored to those through other paths previously proposed ${ }^{1-3}$. The graphite-to-diamond transformation is governed by the formation of nanoscale coherent interfaces (diamond nucleation), which, under static compression, advance to consume the remaining graphite (diamond growth). These results also shed light on transformation mechanisms of other carbon materials and boron nitride under different synthetic conditions.

\section{Main Text}

Carbon exhibits numerous allotropes due to its ability to form various bonds through orbital hybridization. Among all the allotropes, graphite and diamond (with $s p^{2}$ and $s p^{3}$ hybridization, respectively) are the most ubiquitous and have been extensively exploited by humans for several millennia. While both occurring in nature, synthesis of diamond from graphite was not successful until the middle of last century ${ }^{6,7}$. The transformation from graphite to diamond can be made under different synthetic conditions, such as high pressure high temperature (HPHT) with ${ }^{6}$ or without catalyst ${ }^{7,8}$, explosive shock ${ }^{9}$, and even low-temperature compression under severe shear deformation ${ }^{10}$. Along with these experimental efforts, understanding the transformation from graphite to diamond has attracted broad attention but remained a significant challenge ${ }^{11}$.

Largely based on diffraction data from recovered samples, several concerted transformation mechanisms were proposed to account for the graphite-to-diamond transformation ${ }^{1,2}$. In hexagonal graphite $(\mathrm{HG})$, graphene layers are arranged in AB-type stacking, with carbon atoms in each layer bonded covalently in a honeycomb-like lattice via $s p^{2}$ hybridization. According to the concerted transformation mechanisms, $\mathrm{HG}$ undergoes several possible variations in stacking order to transform into cubic diamond (CD) or hexagonal diamond (HD) where all carbon atoms are bonded covalently by $s p^{3}$ hybridization. The $A B$ stacking may change into $A B C$ stacking, followed by collective puckering to transform into $C D^{2}$. Alternatively, the $A B$ stacking may change either to $A A$ stacking followed by puckering to transform into $H D$, or to $A B C$ stacking followed by puckering to transform into $C D$ or buckling to transform into HD. Some reports, again largely based on diffraction, suggested that formation of HD is energetically favored at lower synthesis temperatures ${ }^{12}$. This prompted nucleation-and-growth models $s^{3,13}$ with two types of transient heterophase junctions proposed between diamond nuclei and graphite matrix ${ }^{11,14}$ : One is a 
graphite-diamond diphase connected with weak van der Waals interaction, the other is covalently bonded interfaces between diamond and graphitic domains with a reduced interlayer distance less than 2.5 angstrom. Similar to the nucleation-and-growth mechanisms, a wave-like lattice buckling and slipping model suggested a stacking order change from $A B$ to $A B C$ by bending graphitic layers, followed by formation of transient heterophase junctions to complete the transformation to $C D^{15}$.

Despite the numerous mechanisms proposed, the graphite-to-diamond transformation process remains elusive. The main obstacle to understanding the transformation is that the process occurs under HPHT without in situ information, particularly at atomic scale. Postmortem examinations on the structure of graphite recovered from HPHT synthesis typically rely on X-ray diffraction (XRD), which is insensitive to small amounts of defects or intermediate phases in the sample. In the absence of microscopic information, interpretation of the XRD data is sometimes nonunique ${ }^{12,16,17}$. More recently, high-resolution transmission electron microscope (HRTEM) has been applied to natural and laboratory-shocked samples, revealing a diamond-graphite hybrid structure composed of graphitic layers inserted within Pandey $(2 \times 1)$ reconstructed surfaces of diamond planes ${ }^{4,5}$. The proposed crystal structure gives rise to diffraction peaks resembling those of graphite (with an interlayer spacing of $3.0 \AA$ ) and diamond. Although the origin of this hybrid structure and its correlation with the graphite-to-diamond transformation remains unclear ${ }^{4,5}$, the idea of hybrid structure provides an alternative view of the reported "compressed graphite" with a $3.1 \AA$ Anterlayer spacing ${ }^{12,18-22}$, and may play an important role in understanding the graphite-todiamond transformation.

In this study, we investigate graphite samples recovered from static HPHT synthesis with state-of-the-art scanning transmission electron microscopy (STEM). Partially transformed samples are characterized by graphite and diamond nanodomains interlocked via coherent interfaces. The graphite domains, with interlayer spacings centering at ca. 3.1 $\AA$, are intimately connected to diamond domains with numerous stacking faults. Atomic-resolution high-angle annular dark-field (HAADF) STEM observations reveal four basic structural motifs constituting the graphite-diamond interfaces. Theoretical calculations suggest a progressive graphite-to-diamond transformation process characterized by formation of graphite-diamond interfaces and subsequent advance of the interfaces for diamond growth, consistent with experimental observations. This work thus clarifies the long-standing puzzle ever since the first successful static synthesis of diamond.

Selected XRD patterns of graphite samples recovered from $15 \mathrm{GPa}$ and temperatures between $1200^{\circ} \mathrm{C}$ and $2000^{\circ} \mathrm{C}$ are shown in Fig. $1 \mathrm{a}$, along with the pristine graphite whose strong and sharp (00/) peak indicates excellent crystallinity. After HPHT treatment, the main diffraction peaks are consistent with those previously observed in graphite compressed at moderate temperatures ${ }^{12}$, where peaks not belonging to CD were attributed to the so-called "compressed graphite" (3.1 and $1.55 \AA$ ) and HD (2.17 and $1.16 \AA$ ). Such assignments, however, are under debate ${ }^{12,23}$. With increasing synthesis temperature and under identical heating duration, intensities of diffraction peaks from CD increase, while the other peaks gradually diminish. A kinetic phase diagram is constructed based on XRD measurements, as 
shown in Fig. 1b. Graphite remains unchanged in low-temperature $\left(T<900^{\circ} \mathrm{C}\right)$ and low-pressure $(\mathrm{P}<10$ $\mathrm{GPa}$ ) regions. Above $900^{\circ} \mathrm{C}$ and $10 \mathrm{GPa}$, a multi-phase region emerges (orange field), where $\mathrm{CD}$ coexists with other metastable carbon phases such as "compressed graphite". At sufficiently high temperatures and pressures, the recovered samples are predominantly CD (light blue field). The well-established equilibrium phase boundary between graphite and diamond is drawn as the dashed line ${ }^{24}$.

Detailed TEM observations on quenched samples provide direct insight into the mechanism of graphiteto-diamond transformation under static compression. Extended Data Figure 1 shows typical microstructures of samples recovered from $15 \mathrm{GPa}$ and various temperatures. All recovered samples are composed of diamond and (compressed) graphite. Fraction of the graphitic phase decreases monotonically with increasing synthesis temperature, consistent with the diminishing diffraction peak at $3.1 \AA$ (Fig. 1a). Figure 2a is a bright field (BF) STEM image from a sample recovered from $15 \mathrm{GPa}$ and $1200^{\circ} \mathrm{C}$, in which diamond (D) and graphite (G) nanodomains are clearly distinguished. In neighboring diamond and graphite domains, the lattice fringes of the two phases are tilted relative to one another, forming interfaces different from the $(113)_{C D}$ or $(111)_{C D}$ types as previously proposed for meteoritic or laboratory-shocked diamonds based on TEM observations $s^{4,5,11,14,25}$. High-resolution HAADFSTEM observations further confirm the tightly bonded graphitic and diamond domains (Fig. 2b). The graphite domains show a reduced interlayer spacing of ca. 3.1 $\AA$, and the lattice fringes are distorted, especially adjacent to the interfaces. The diamond domains exhibit considerable stacking disorder in the close-packed carbon bilayers. Magnified HAADF-STEM images in Fig. $2 \mathrm{c}$ and $2 \mathrm{~d}$ reveal a remarkable oneto-one correspondence between atomic layers in graphite and kinked carbon bilayers in diamond. Hereafter, this unique hybrid carbon, which consists of nanoscale graphite and diamond units bonding each other through coherent interfaces, is referred to as Gradia. The corresponding interface is referred to as gradia interface. The phase/microstructure evolution of graphite under different P-T conditions (Fig. $1 \mathrm{~b}$ and Extended Data Fig. 1) and the newly discovered gradia interfaces suggest that the formation and migration of the interfaces play a decisive role in graphite-to-diamond transformation under static pressure: Diamond growth is accomplished by advancing the interfaces into graphite.

Examples of HAADF-STEM images of gradia interfaces are shown in Fig. 3 and Extended Data Fig. 2. The graphite and diamond domains exhibit the following orientation relations: or , with no definitive epitaxial relationship across the interface. Based on the HAADF-STEM observations, four primary structural motifs are identified to constitute the gradia interfaces, as shown in Fig. $3 \mathrm{c}$ where the corresponding puckering and buckling processes in graphitic layers with different stacking orders are indicated by red arrows. When viewed along, the and planes form a rhombic pattern with equal side-length of $2.18 \AA . A$ rhombus in $\mathrm{CD}$ can connect to the (001) lattice of compressed graphite through a vertex with either an obtuse or an acute angle, forming two structural motifs which are referred to as Gradia-CO and Gradia-CA, respectively. Similarly, when viewed along, the and planes form a rectangular pattern with two sidelengths of 2.18 and $2.06 \AA$, respectively. The adjacent (001) layers of compressed graphite can either buckle into a boat conformation and transform into with a $d$-spacing of $2.18 \AA$, or pucker into a chair 
conformation and transform into with a $d$-spacing of $2.06 \AA$. These two structural motifs are referred to as Gradia-HB and Gradia-HC, respectively.

Under HPHT, atomic layers in graphite endure compression, bending and interlayer sliding, resulting in highly localized variations in interlayer distance, curvature and stacking order, which may induce new bonding across neighboring graphite layers to form different interface structures. The gradia interface constituted with aforementioned structural motifs has great variability and flexibility to accommodate such local structural variations (Fig. 3c). Note that while both Gradia-HB and Gradia-HC can coexist with Gradia-CO and Gradia-CA, Gradia-HB and Gradia-HC are mutually excluding (Fig. 3 and Extended Data Fig. 2). This is because a plane cannot be completely filled by two differently oriented rectangles, with all vertices overlapping. Under HPHT conditions, gradia interfaces advance into graphite, promoting diamond growth. For example, Extended Data Figure 3 shows schematically the advance of the Gradia$\mathrm{CO}$ and Gradia-HC interfaces (Fig. 3c) into graphite, with several new motifs forming at the frontline. Similar growth processes also occur for other gradia interfaces with different combinations of structural motifs. As the interface advances to the graphite side, the specifically combined structural motifs impose constraints on the bonding of carbon atoms in adjacent graphite layers, resulting in significant stacking disorder of carbon bilayers in the as-grown diamond (Fig. 3 and Extended Data Fig. 2). It may be worth noting that STEM observations did not identify any pure HD domains in the recovered samples, even though the XRD patterns show a prominent peak at $2.17 \AA$ and two weaker ones at 1.93 (shoulder) and $1.16 \AA$, which were previously attributed to $H D^{12}$. Actually, all diamond domains are characterized by high density of stacking faults. Similar hexagonal-cubic stacking disorders also exist in natural and laboratory-shocked diamonds ${ }^{23}$, and account for the hexagonal feature in diffraction patterns ${ }^{16,17}$. One thus should exercise caution when claiming new diamond phases. This potential ambiguity does not preclude the existence of HD though. For example, we did observe an HD nanodomain, $3 \mathrm{~nm}$ in thickness and $30 \mathrm{~nm}$ laterally from HPHT-treated carbon onions ${ }^{26}$. Larger HD phases may be produced with carefully selected carbon precursors and fine-tuned P-T conditions.

To understand the origin of gradia interfaces and their roles in graphite-to-diamond transformation, we conducted first-principles calculations on intentionally designed hybrid crystals with characteristic gradia interfaces shown in Fig. 3c (see Methods, Extended Data Fig. 4 and Extended Data Table 1 for more details). As shown in Extended Data Fig. 4, the unit cell for each hypothetic crystal is separated into $s p^{2}$ hybridized graphitic (gray-colored atoms) and $s p^{3}$-hybridized diamond (gold-colored atoms) sections that are bonded coherently via a gradia interface (green-colored atoms). The thermodynamic, dynamic, and mechanical stabilities of these crystal structures are shown in Extended Data Fig. 5 and Extended Data Table 2. Transformation energy barriers from graphite to diamond through these intermediate crystal structures were evaluated under pressure at $0 \mathrm{~K}$ with variable-cell nudged-elastic-band (VCNEB) simulation method ${ }^{27,28}$ as implemented in the USPEX code ${ }^{29,30}$. The transformation processes are summarized in Fig. 4 and Extended Data Fig. 6. The energy barriers required to form gradia interfaces directly from graphite are all higher than those for diamond growth by advancing the gradia interfaces into graphite (Fig. 4a). Note that in all considered cases, the energy barriers decrease 
monotonically with increasing pressure in the range of 0-15 GPa (Fig. 4b and 4c), and the transformation barriers along the pathways through the gradia interfaces are substantially lower than those along classic concerted transformation pathways ${ }^{27}$.

Figure 4d-e and Extended Data Fig. 6 provide atomistic snapshots from pure graphite to diamond under $10 \mathrm{GPa}$ through hypothetic crystals with different gradia interfaces (See Supplementary Videos 1-4 for the whole processes). During the transformation, graphite layers undergo wave-like bending with remarkable localized variations in stacking order and interlayer spacing, inducing additional bonding across adjacent graphite layers to form gradia interfaces in regions with suitable stacking order and interlayer spacing. For example, Figure $4 \mathrm{~d}$ shows five snapshots of the transformation from $\mathrm{HG}$ to $\mathrm{CD}$. The oscillation of the graphitic layers (second snapshot from the top) results in localized changes of stacking order from $A B$ to $C B A$ companied by reduced interlayer spacing, leading to the formation of a Gradia-CO interface and the appearance of the first diamond-like bonding. At this diamond nucleation stage, the energy barrier reaches the maximum (Fig. 4a). The gradia interface then advances from both sides into graphitic sections, resulting in the growth of diamond lattice (third and fourth snapshots) until the transformation to CD is complete (fifth snapshot). In contrast, previously proposed wave-like buckling and slipping mechanism invokes uniform interlayer distances without forming gradia interfaces ${ }^{15}$. Figure 4 a suggests that once a gradia interface is formed, further formation of diamond is energetically favored even under metastable conditions. This is confirmed by in-situ STEM observation (Extended Data Fig. 7). Under electron beam irradiation in vacuum, new diamond-like atomic bonding is identified from the graphite side of Gradia-CO interface. This remarkable observation is due to the lower energy barrier for diamond growth via step-by-step advancing of the gradia interface.

By integrating $s p^{2}$-hybidized graphite and $s p^{3}$-hybridized diamond nanodomains with strong coherent interfaces, Gradia has the prospect of combining both parties' advantages, with potentially a wide range of properties for multifunctional applications ${ }^{4}$. The gradia interfaces may also play a significant role in tuning material properties. For example, the designed hybrid crystals display obvious metallicity (Extended Data Fig. 4), contributed mostly by atoms in the graphitic section and gradia interface. Note that the contribution to the metallicity from interface atoms is comparable with, or even higher than, that from graphitic atoms in Gradia-CO and Gradia-HB crystals, due to the presence of $s p^{2}$-hybridized atoms (circled in red) at the interface. In Gradia, the proportion-tunable graphite and diamond domains together with the versatile gradia interfaces offer additional freedom in engineering nanostructures, for desired properties. Specifically, differently hybridized carbon atoms in Gradia contribute to different functionalities, e.g., $s p^{3}$ atoms to superhardness, $s p^{2}$ atoms to electrical conductivity, and $s p^{2}-s p^{3}$ mixed atoms near the interfaces to toughness ${ }^{4}$. With regulated fractions and distributions of different types of atoms, a variety of properties, which are inaccessible for diamond and graphite separately, may be tailored for Gradia.

The transformation from graphite to diamond under static compression occurs in two stages, i.e., the formation of coherent gradia interface (diamond nucleation) and subsequently the advance 
of the interface (diamond growth). The transformation mechanism clarified in this work can serve as a guidance in understanding transformations of boron nitride and other carbon phases such as carbon onions under high pressure. Beyond the transformation mechanism, the newly unveiled Gradia marks a major step towards nanostructure and properties engineering in diamond-related materials, and provides new opportunities in pursuing desired combination of mechanical and electronic properties, such as simultaneous superhardness, high toughness and electrical conductivity. (2438 words)

Online Content Methods, along with any additional Extended Data display items and Source Data, are available in the online version of the paper; references unique to these sections appear only in the online paper.

\section{References}

1 Fahy, S., Louie, S. G. \& Cohen, M. L. Theoretical total-energy study of the transformation of graphite into hexagonal diamond. Phys. Rev. B 35, 7623-7626 (1987).

2 Scandolo, S., Bernasconi, M., Chiarotti, G. L., Focher, P. \& Tosatti, E. Pressure-induced transformation path of graphite to diamond. Phys. Rev. Lett. 74, 4015-4018 (1995).

3 Khaliullin, R. Z., Eshet, H., Kuhne, T. D., Behler, J. \& Parrinello, M. Nucleation mechanism for the direct graphite-to-diamond phase transition. Nat. Mater. 10, 693-697 (2011).

4 Németh, P. et al. Complex nanostructures in diamond. Nat. Mater. 19, 1126-1131 (2020).

5 Nemeth, P. et al. Diamond-graphene composite nanostructures. Nano Lett. 20, 3611-3619 (2020).

6 Bundy, F. P., Hall, H. T., Strong, H. M. \& Wentorfjun, R. H. Man-made diamonds. Nature 176, 51-55 (1955).

7 Bundy, F. P. Direct conversion of graphite to diamond in static pressure apparatus. Science 137, 1057-1058 (1962).

8 Irifune, T., Kurio, A., Sakamoto, S., Inoue, T. \& Sumiya, H. Ultrahard polycrystalline diamond from graphite. Nature 421, 599-600 (2003).

9 Decarli, P. S. \& Jamieson, J. C. Formation of diamond by explosive shock. Science 133, 1821-1822 (1961).

10 Dong, J. et al. Decompression-induced diamond formation from graphite sheared under pressure. Phys. Rev. Lett. 124, 065701 (2020).

11 Zhu, S.-c., Yan, X.-Z., Liu, J., Oganov, A. R. \& Zhu, Q. A revisited mechanism of the graphite-todiamond transition at high temperature. Matter 3, 864-878 (2020). 
12 Bundy, F. P. \& Kasper, J. S. Hexagonal diamond-a new form of carbon. J. Chem. Phys. 46, 34373446 (1967).

13 Britun, V. F., Kurdyumov, A. V. \& Petrusha, I. A. Diffusionless nucleation of lonsdaleite and diamond in hexagonal graphite under static compression. Powder Metall. Met. Ceram. 43, 87-93 (2004).

14 Xie, Y. P., Zhang, X. J. \& Liu, Z. P. Graphite to diamond: Origin for kinetics selectivity. J. Am. Chem. Soc. $139,2545-2548(2017)$.

15 Xie, H., Yin, F., Yu, T., Wang, J. T. \& Liang, C. Mechanism for direct graphite-to-diamond phase transition. Sci. Rep. 4, 5930 (2014).

16 Salzmann, C. G., Murray, B. J. \& Shephard, J. J. Extent of stacking disorder in diamond. Diam. Relat. Mater. 59, 69-72 (2015).

17 Murri, M. et al. Quantifying hexagonal stacking in diamond. Sci. Rep. 9, 10334 (2019).

18 Alvarez-Murga, M. et al. "Compressed graphite" formed during $\mathrm{C}_{60}$ to diamond transformation as revealed by scattering computed tomography. Phys. Rev. Lett. 109, 025502 (2012).

$19 \mathrm{Hu}, \mathrm{M}$. et al. Compressed glassy carbon: An ultrastrong and elastic interpenetrating graphene network. Sci. Adv. 3, e1603213 (2017).

20 Irifune, T. et al. Formation of pure polycrystalline diamond by direct conversion of graphite at high pressure and high temperature. Phys. Earth Planet. Inter. 143-144, 593-600 (2004).

21 Niwase, K. et al. Quenchable compressed graphite synthesized from neutron-irradiated highly oriented pyrolytic graphite in high pressure treatment at $1500^{\circ} \mathrm{C}$. J. Appl. Phys. 123, 161577 (2018).

22 Yang, X. et al. Diamond-graphite nanocomposite synthesized from multi-walled carbon nanotubes fibers. Carbon 172, 138-143 (2021).

23 Nemeth, P. et al. Lonsdaleite is faulted and twinned cubic diamond and does not exist as a discrete material. Nat. Commun. 5, 5447 (2014).

24 Bundy, F. P. et al. The pressure-temperature phase and transformation diagram for carbon; updated through 1994. Carbon 34, 141-153 (1996).

25 Garvie, L. A. J., Nemeth, P. \& Buseck, P. R. Transformation of graphite to diamond via a topotactic mechanism. Am. Mineral. 99, 531-538 (2014).

26 Yue, Y. H. et al. Hierarchically structured diamond composite with exceptional toughness. Nature 582, 370-374 (2020). 
27 Dong, X. et al. An ab initio study on the transition paths from graphite to diamond under pressure. J. Phys. Condens. Matter. 25, 145402 (2013).

28 Qian, G.-R. et al. Variable cell nudged elastic band method for studying solid-solid structural phase transitions. Comput. Phys. Commun. 184, 2111-2118 (2013).

29 Glass, C. W., Oganov, A. R. \& Hansen, N. Uspex-evolutionary crystal structure prediction. Comput. Phys. Commun. 175, 713-720 (2006).

30 Lyakhov, A. O., Oganov, A. R., Stokes, H. T. \& Zhu, Q. New developments in evolutionary structure prediction algorithm uspex. Comput. Phys. Commun. 184, 1172-1182 (2013).

\section{Declarations}

Acknowledgments This work is supported by National Natural Science Foundation of China (Nos. 52090020, 91963203, 51772260, U20A20238, 52025026, 52073245, 51722209, 51525205), and the National Key R\&D Program of China (2018YFA0703400 and 2018YFA0305900). Z.Z. acknowledges the NSF for Distinguished Young Scholars of Hebei Province of China (E2018203349).

Author contributions Z.Z., B.X. and Y.T. conceived this project; K.L., B.L., D.Y. and Z.Z. prepared the samples; K.L., B.L., Y.G. and M.M. measured XRD; W.H., K.L. and L.S. conducted TEM characterization; K.L., Z.Z. and B.X. determined the Gradia structures; K.L., Y.W., Q.H. and J.H. performed the structural stability calculations; X.D. and X.F.Z. performed the transition pathway calculations; K.L., Z.Z., B.X., Y.W., Y.T., Z.L. and Y.Z. analyzed the data; K.L., Z.Z., B.X., Y.W. and Y.T. drafted the manuscript with contributions from all authors. K.L., B.L., W.H. and X.D. contributed equally to this work.

Author Information Reprints and permissions information is available at www.nature.com/reprints. The authors declare no competing financial interests. Readers are welcome to comment on the online version of the paper. Publisher's note: Springer Nature remains neutral with regard to jurisdictional claims in published maps and institutional affiliations. Correspondence and requests for materials should be addressed to Z.Z. (zzhao@ysu.edu.cn)

\section{Competing interests}

The authors declare that they have filed a provisional patent application in Aug. 2019.

\section{Methods}

Sample synthesis. The gradia-containing samples were synthesized from graphite $(99.99 \%$, Alfa Aesar) under conditions of $10-25 \mathrm{GPa}$ and $1000-2300^{\circ} \mathrm{C}$. HPHT experiments were performed with a 10-MN double-stage large-volume multi-anvil system by using standard COMPRES 10/5 (or 8/3) sample assembly consisting of a $10-\mathrm{mm}$ (or 8-mm) spinel $+\mathrm{MgO}$ octahedron with a Re heater and a $\mathrm{LaCrO}_{3}$ thermal insulator. Temperature was measured with Type $C$ thermocouples, and pressure estimated from 
previously determined calibration curves. During the synthesis, pressure increased at $2 \mathrm{GPa}$ per hour to the target pressure, then the sample was heated with a rate of $20^{\circ} \mathrm{C}$ per minute to the target temperature. In all the experiments, the sample was maintained under the target pressure and temperature for 2 hours. After that, was dropped to room temperature at $50^{\circ} \mathrm{C}$ per minute, followed by pressure release with a rate of $2 \mathrm{GPa}$ per hour. The recovered sample rods were $1-2.5 \mathrm{~mm}$ in diameter and height.

Ultra-thin TEM sample preparation. To eliminate grain overlaps in STEM imaging, foils with thickness of about $60 \mathrm{~nm}$ were cut with focus ion beam (FEl Helios 5 CX DualBeam), and further thinned to $20 \mathrm{~nm}$ with low energy Ar-ion milling (Fischione Model 1040 NanoMill). Before loading into the microscope, the foils were cleaned with $\mathrm{H}_{2} / \mathrm{O}_{2}$ plasma (Gatan 695 Plasma cleaner) for 40 s to eliminate possible carbon contamination.

HAADF-STEM measurement. STEM measurements were conducted with a spherical aberration corrected scanning transmission electron microscope (FEI Themis Z), with a monochromator, operating at an accelerating voltage of $300 \mathrm{kV}$. BF, ADF and HAADF images were obtained by combining 20 frames from acquired series with drift correction (DCFI in software of Velox, Thermo Fisher). The probe convergence angle was set at $25 \mathrm{mrad}$. Collecting angles of BF and ADF were set at $6 \mathrm{mrad}$ and 16-62 mrad, respectively. The collecting angle of HAADF was set at 65-200 mrad to eliminate effects of coherent scattering.

X-ray diffraction. Powder X-ray diffraction patterns of recovered samples were collected using Bruker D8 Discover $\left(\mathrm{Cu} \mathrm{K}_{a}\right)$.

DFT simulation. The hypothetic crystal structures based on gradia interfaces were constructed with the Materials Visualizer module in Materials Studio (Accelrys Software Inc.). The calculations were performed at $0 \mathrm{~K}$ based on the density functional theory (DFT) as implemented in the CASTEP code ${ }^{31}$, and the ultrasoft pseudopotentials were used ${ }^{32,33}$. The local density approximation (LDA) exchangecorrelation functional of Ceperley and Alder parameterized by Perdew and Zunger (CA-PZ) was utilized for structural optimization and calculations of total energies, elastic properties and phonon spectra ${ }^{34,35}$. A $k$-point sampling ${ }^{36}$ of $2 \pi \times 0.03 \AA^{-1}$ and a plane-wave cutoff of $600 \mathrm{eV}$ were used. The selected calculation parameters were all tested to ensure that energy convergence was less than $1 \mathrm{meV}$ per atom. For comparison, we also performed similar calculations on pure graphite and diamond crystals. To reveal the transformation mechanism from graphite to diamond via the gradia interfaces, we performed variable-cell nudged-elastic-band (VCNEB) simulations ${ }^{27,28}$ at $0,5,10$ and $15 \mathrm{GPa}$, as implemented in the USPEX code ${ }^{29,30}$. Both initial and final states were relaxed at set pressures. Then, the initial pathways were subsequently refined by the VCNEB method and optimized to find the minimum energy pathways. The forces and stresses were computed by VASP $\operatorname{code}^{37}$ with the LDA exchange-correlation functional of $\mathrm{CA}-\mathrm{PZ}^{34}$. The projector augmented-wave (PAW) method was adopted, with $2 s^{2} 2 p^{2}$ treated as valence electrons for $C$ atom. Plane-wave cutoff energies were $600 \mathrm{eV}$ and $k$-point meshes were sampled with the resolution of $2 \pi \times 0.04 \AA^{-1}$. Spring constants were set as $40 \mathrm{eV} / \AA^{2}$. The climbing image technique ${ }^{38}$ was 
used to precisely locate transition states after hundreds of VC-NEB steps. For the mechanical stability, the 13 independent elastic constants $C_{\text {ij }}$ for a stable monoclinic structure should satisfy the Born stability criteria $^{39-42}$.

Data availability. The data that support the findings of this study are available from corresponding authors on reasonable request.

31 Clark, S. J. et al. First principles methods using CASTEP. Zeitschrift für Krist. - Cryst. Mater. 220, 567-570 (2005).

32 Laasonen, K., Car, R., Lee, C. \& Vanderbilt, D. Implementation of ultrasoft pseudopotentials in ab initio molecular dynamics. Phys. Rev. B 43, 6796-6799 (1991).

33 Vanderbilt, D. Soft self-consistent pseudopotentials in a generalized eigenvalue formalism. Phys. Rev. B 41, 7892-7895 (1990).

34 Perdew, J. P. \& Zunger, A. Self-interaction correction to density-functional approximations for many-electron systems. Phys. Rev. B 23, 5048-5079 (1981).

35 Ceperley, D. M. \& Alder, B. J. Ground State of the Electron Gas by a Stochastic Method. Phys. Rev. Lett. 45, 566-569 (1980).

36 Monkhorst, H. J. \& Pack, J. D. Special points for Brillouin-zone integrations. Phys. Rev. B 13, 51885192 (1976).

37 Kresse, G. \& Furthmuller, J. Efficient iterative schemes for ab initio total-energy calculations using a plane-wave basis set. Phys. Rev. B 54, 11169-11186 (1996).

38 Henkelman, G., Uberuaga, B. P. \& Jónsson, H. A climbing image nudged elastic band method for finding saddle points and minimum energy paths. J. Chem. Phys. 113, 9901-9904 (2000).

39 Mouhat, F. \& Coudert, F.-X. Necessary and sufficient elastic stability conditions in various crystal systems. Phys. Rev. B 90, 224104 (2014).

40 Born, M., Huang, K. \& Lax, M. Dynamical Theory of Crystal Lattices. Am. J. Phys. 23, 474-474 (1955).

41 Hill, R. The Elastic Behaviour of a Crystalline Aggregate. Proc. Phys. Soc. Sect. A 65, 349-354 (1952).

42 Born, M. On the stability of crystal lattices. I. Math. Proc. Cambridge Philos. Soc. 36, 160-172 (1940). 


\section{Figures}
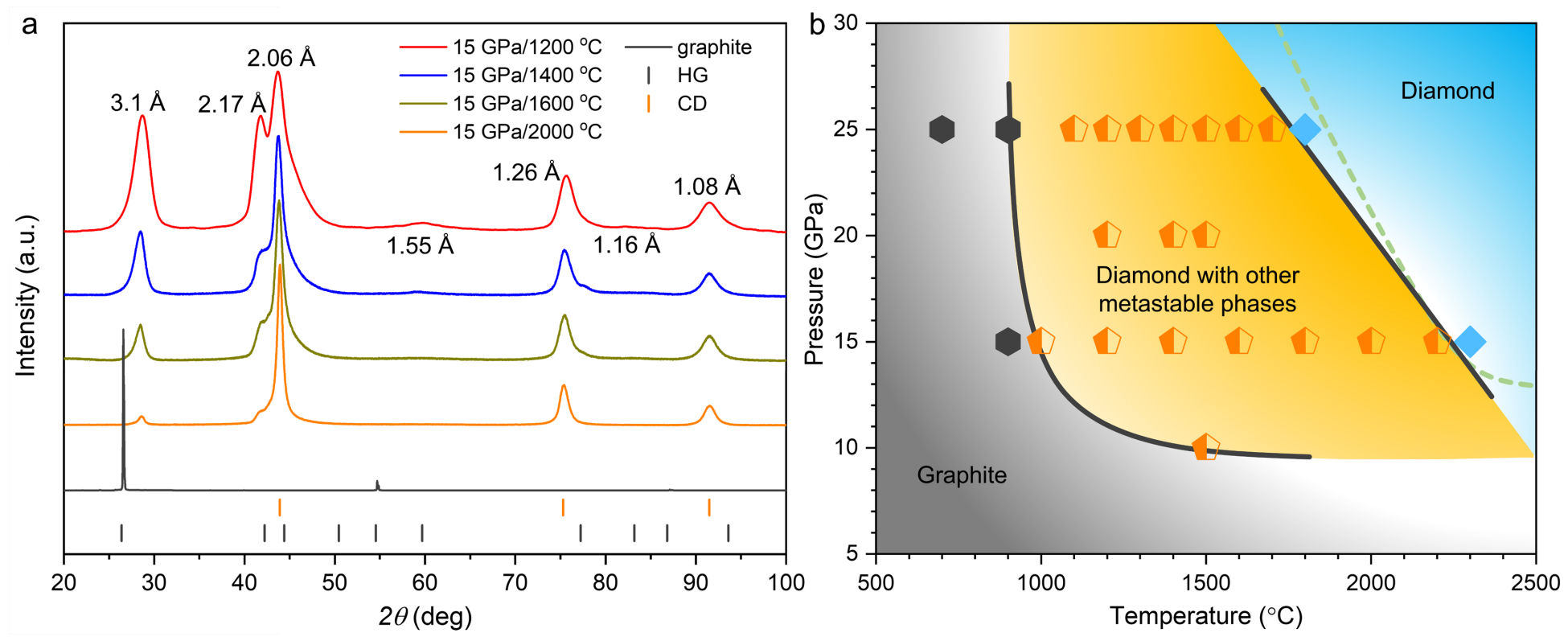

\section{Figure 1}

XRD patterns and phase evolution diagram of graphite under HPHT. a, X-ray diffraction of samples recovered from $15 \mathrm{GPa}$ and $1200^{\circ} \mathrm{C}, 1400^{\circ} \mathrm{C}, 1600^{\circ} \mathrm{C}$, and $2000^{\circ} \mathrm{C}$. The pristine graphite is included for comparison. The colored tags at the bottom indicate standard diffraction lines of graphite (HG) and cubic diamond (CD). b, Kinetic phase diagram of graphite under HPHT determined from XRD results. Hexagons, pentagons, and diamond symbols represent samples that are pure graphite, mixed phases containing CD and other metastable carbon phases, and pure diamond, respectively. Collectively, these data points define three regions as delineated by the solid lines. The dashed line is the established phase boundary between graphite and diamond. 

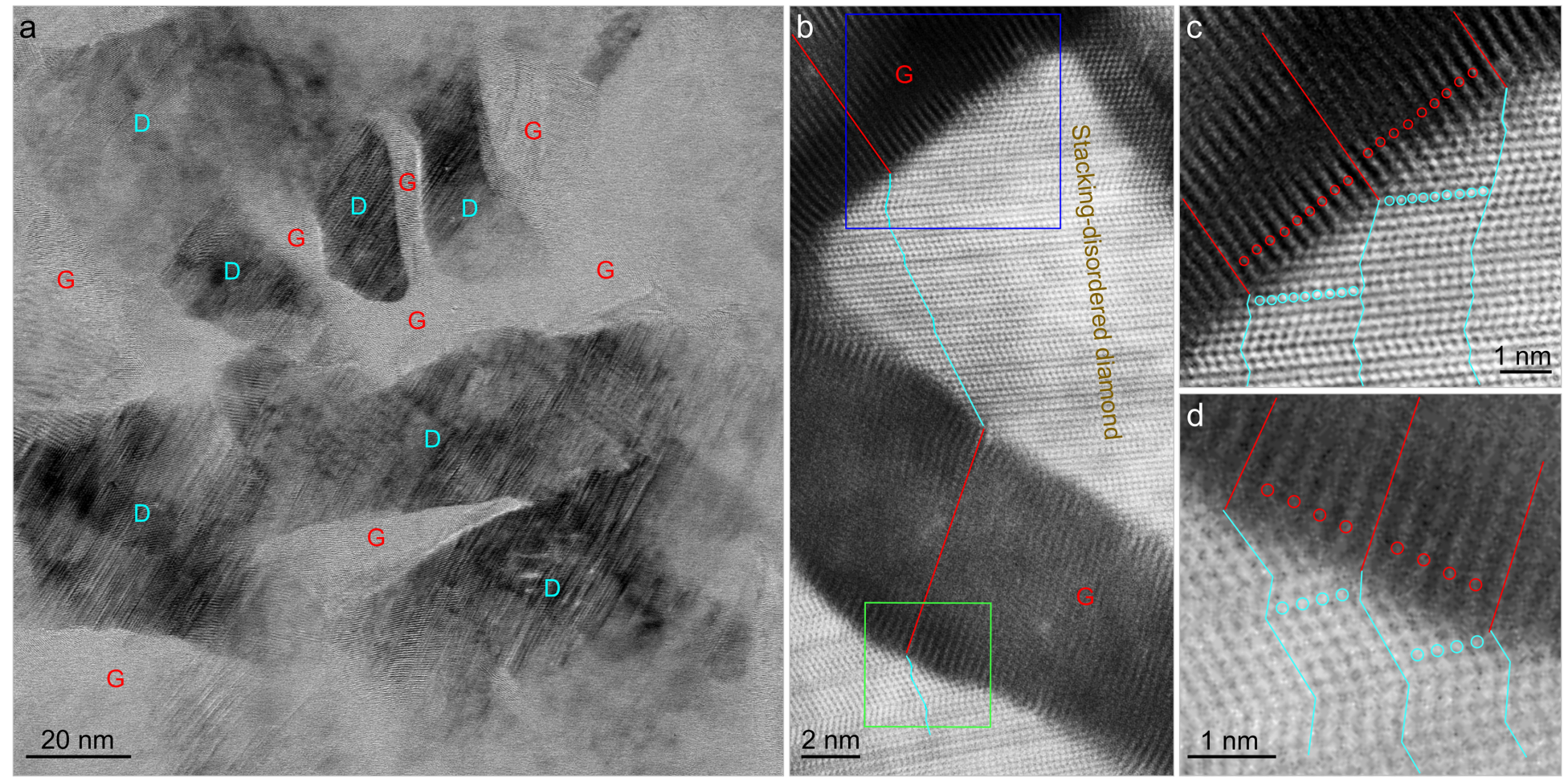

\section{Figure 2}

Microstructures of a sample recovered from $15 \mathrm{GPa}$ and $1200^{\circ} \mathrm{C}$. a, Low-magnification BF-STEM image showing nanoscaled diamond (D) domains embedded in graphite (G). b, High-resolution HAADF-STEM image of graphite domains showing a reduced interlayer spacing of $3.1 \AA$ and diamond domains with numerous stacking faults, with well-defined interfaces between the two phases. Alternating red and cyan lines delineate the end-to-end connectivity between one atomic layer in graphite and kinked carbon bilayer in diamond traversing multiple graphite and diamond domains. $c$ and d, Magnified HAADF-STEM images corresponding to the blue- and green-boxed regions in b, respectively. Red and cyan lines and circles highlight the one-to-one correspondence between the atomic layers in graphite and the kinked carbon bilayers in diamond, respectively. 

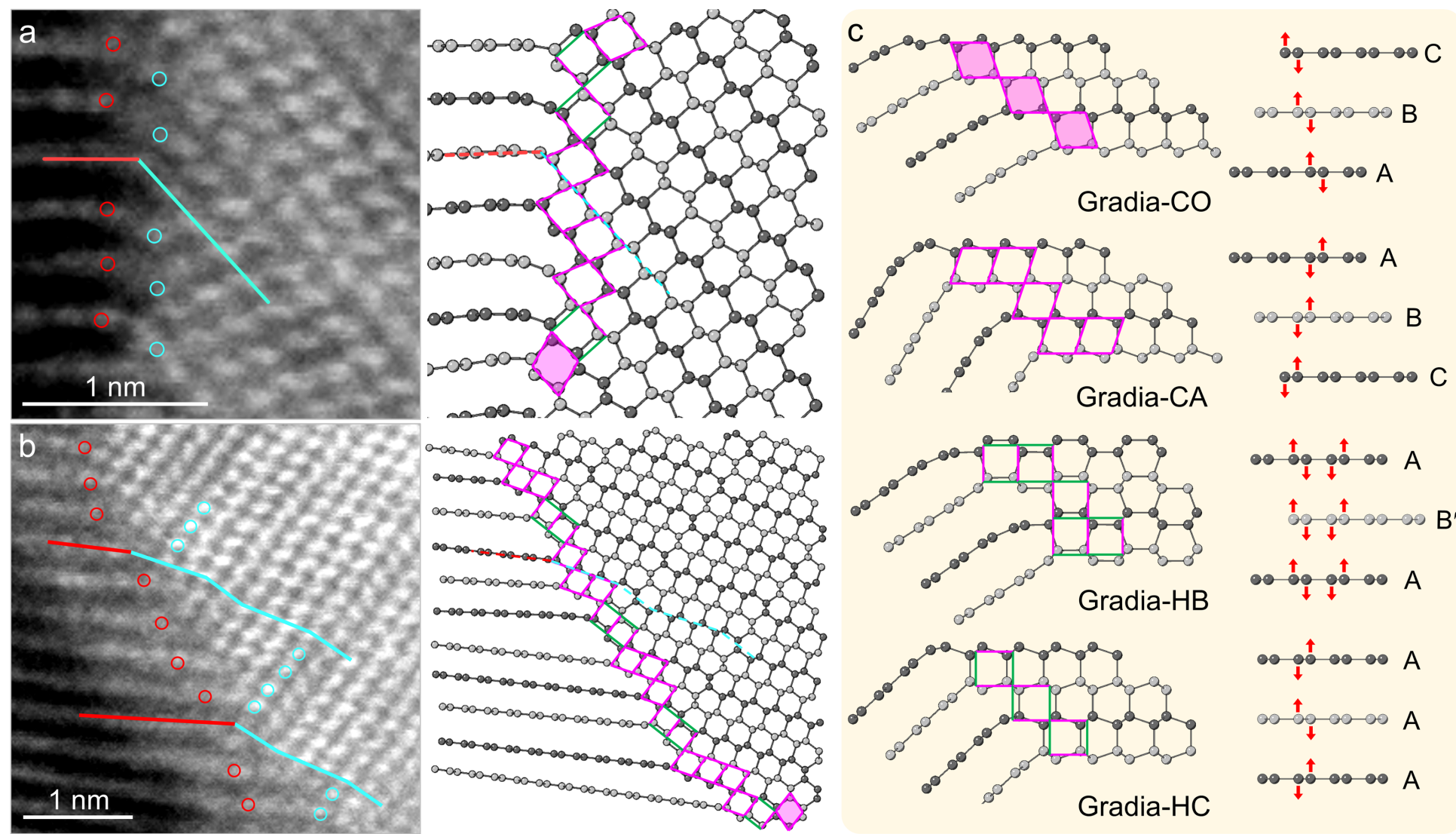

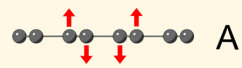

i

๑

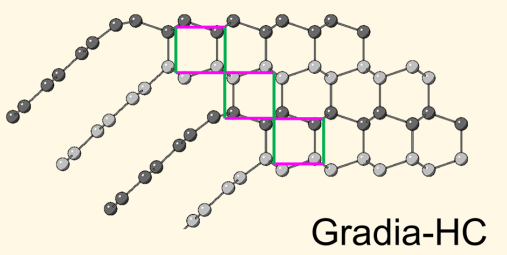

00

A

00 -

Figure 3

Coherent interface structures between graphite and diamond. $a$ and $b$, Atomic-resolution HAADF-STEM images of two gradia interfaces and the corresponding atomic models. Red and cyan lines (circles) delineate the one-to-one correspondence between graphite and diamond. In the atomic models, adjacent graphitic layers are colored with different grayscales for clarity. Structural motifs at the interface are denoted with rhombi (with or without shadows) and rectangles (with different orientations). c, Four representative gradia interfaces. See main text for the details of nomenclature. 

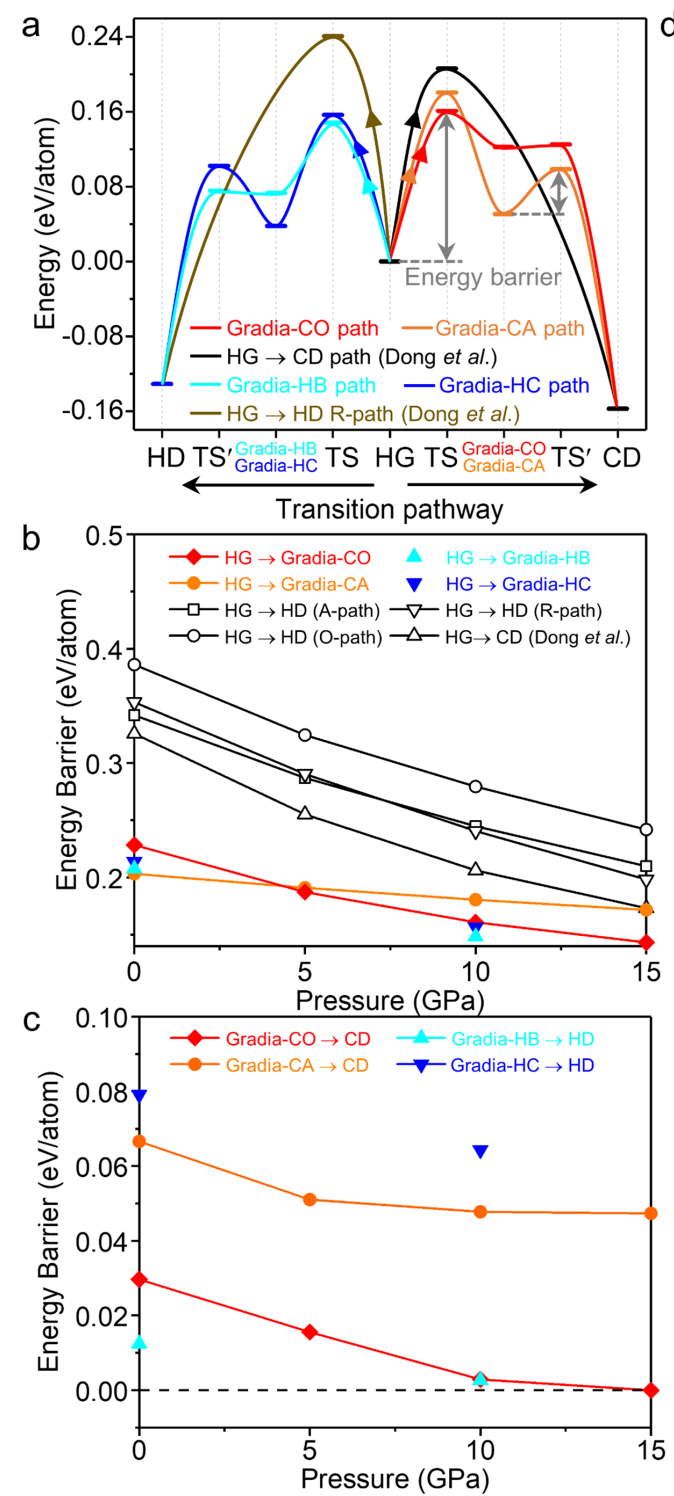

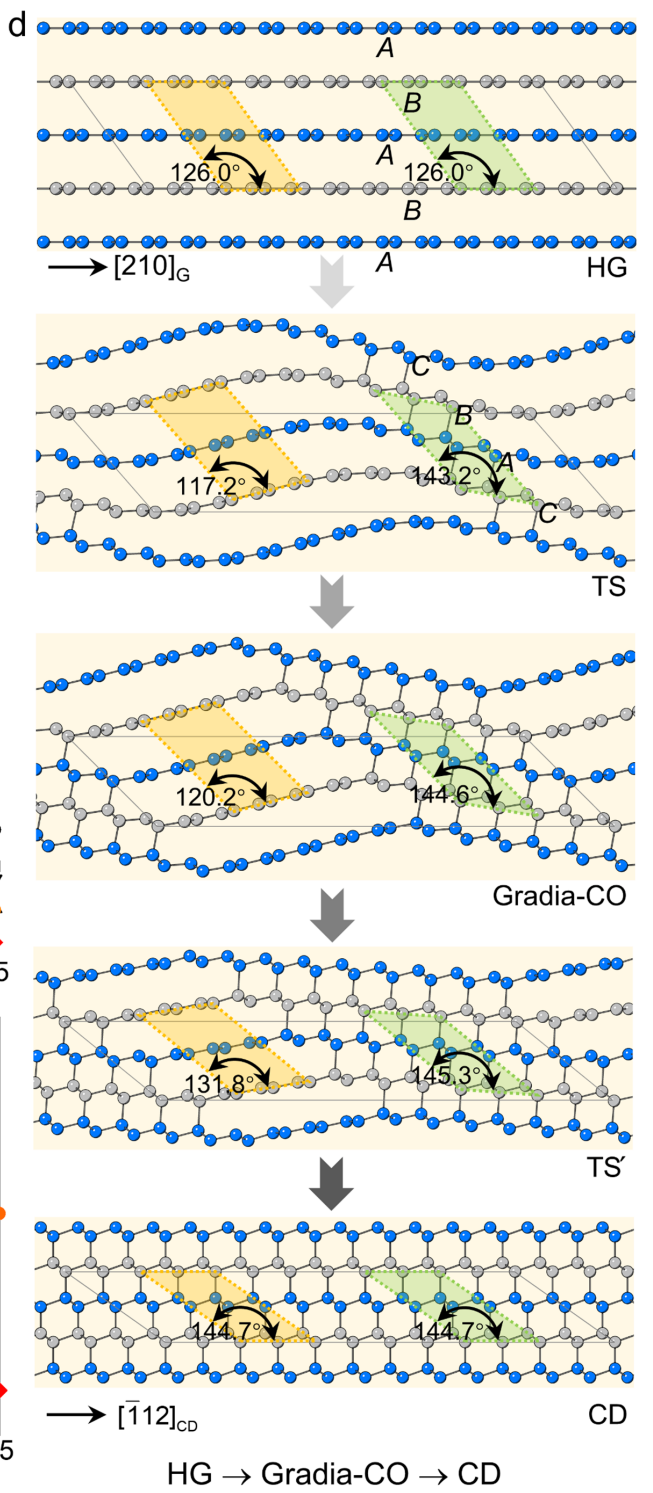

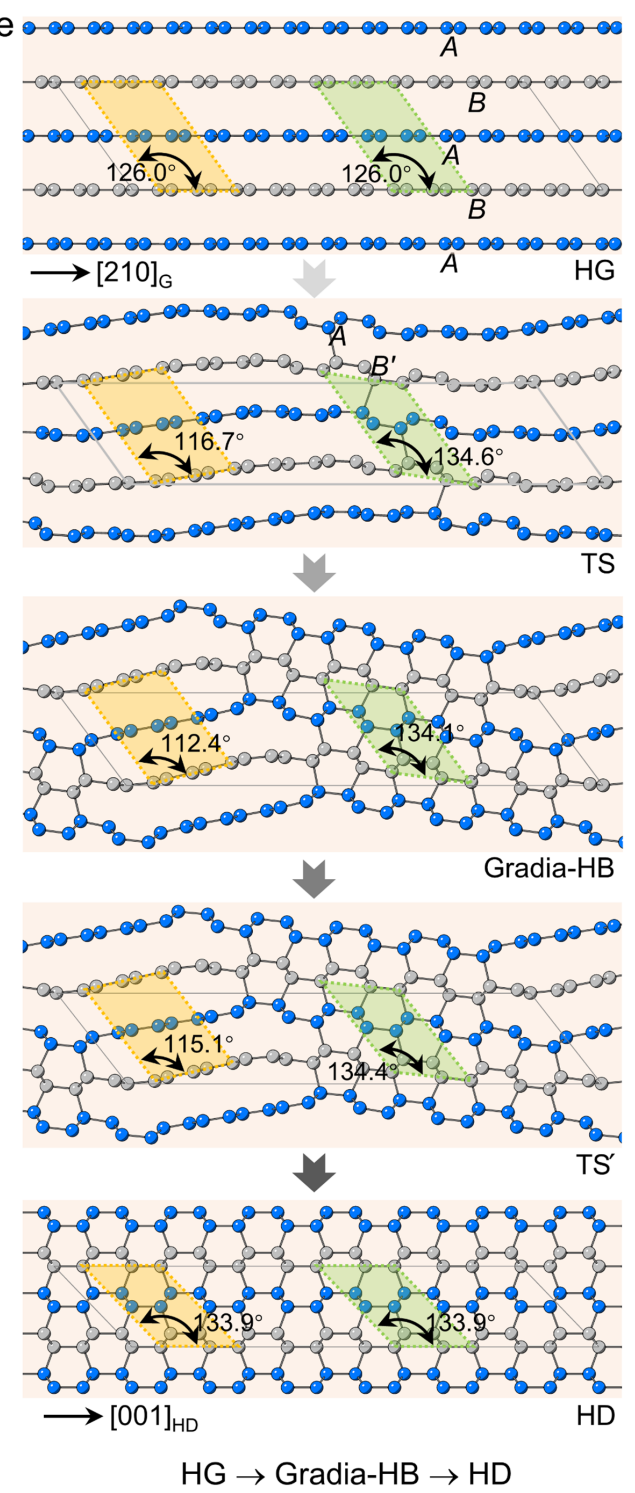

Figure 4

Energy barriers and transformation process from graphite to diamond through intermediate crystals (hypothetic) containing gradia interfaces. a, Energy profile of graphite-to-diamond transformation through different pathways at $10 \mathrm{GPa}$. The maximum energy barrier occurs when the wavy graphitic layers start bonding, i.e., forming the gradia interface along $\mathrm{HG}$ to intermediate crystal. b, Energy barriers decrease with increasing pressure from HG to intermediate crystal. c, Energy barriers decrease with increasing pressure from intermediate crystal to $\mathrm{CD}$ (or HD). The energy barrier in this stage (diamond growth) is significantly lower than that in the nucleation stage (gradia interface formation). Above $10 \mathrm{GPa}$, Gradia$\mathrm{CO}$ and Gradia-HB crystals can convert into diamond with almost no energy barrier. $d$ and e, The atomistic snapshots during graphite-to-diamond transformation through Gradia-CO and Gradia-HB crystals, respectively, under $10 \mathrm{GPa}$. The adjacent graphitic layers are distinguished with gray and blue colors. Under pressure, the graphite layers bend wavily. Bonding across graphite layers starts in the greenshadowed regions with most reduced interlayer spacing of ca. $2.1 \AA$, while the interlayer spacing increase from $2.9 \AA$ to $3.2 \AA$ in the yellow-shadowed regions, leaving graphite stable. Next, the interfaces advance 
gradually into graphite, and diamond nuclei grow into pure diamond eventually. The angles in the greenand yellow-shadowed regions indicate the localized changes in the structure.

\section{Supplementary Files}

This is a list of supplementary files associated with this preprint. Click to download.

- Video1HGCOCD10GPa.mp4

- Video2HGCACD10GPa.mp4

- Video3HGHBHD10GPa.mp4

- Video4HGHCHD10GPa.mp4

- ExtendedData0923.pdf 